\title{
Author Correction: Distinct dynamics and functions of H2AK119ub1 and H3K27me3 in mouse preimplantation embryos
}

Zhiyuan Chen (1), Mohamed Nadhir Djekidel (1) and Yi Zhang (1)

Correction to: Nature Genetics https://doi.org/10.1038/s41588-021-00821-2, published online 5 April 2021.

In the version of this article initially published, the URL in the Code availability section was incorrect. The error has been corrected in the HTML and PDF versions of the article.

Published online: 22 April 2021

https://doi.org/10.1038/s41588-021-00871-6

( ) The Author(s), under exclusive licence to Springer Nature America, Inc. 2021 